\title{
Unexplained Perioperative Hypotension in a Patient Treated with Pine Needle Extract
}

\author{
Heeseung Lee*, Heehun Jeon, Jong In Han, Youn Jin Kim, Chi Hyo Kim, Dong Yeon Kim
}

Department of Anesthesiology and Pain Medicine, School of Medicine, Ewha Womans University, Seoul, South Korea. Email: *leehee@ewha.ac.kr

Received October $29^{\text {th }}, 2012$; revised November $30^{\text {th }}, 2012$; accepted December $12^{\text {th }}, 2012$

\begin{abstract}
Pine needle-based products are popular dietary supplements in East Asia for health promotion. Pine needle extract (PNE) are especially known as potential anti-hypertensive and anti-lipidemic agents. We report a case of profound, long-lasting unexplained hypotension during and after anesthesia in a patient treated with PNE. Bedside transthorasic echocardiography (TTE) was used as a part of useful diagnostic modality.
\end{abstract}

Keywords: Echocardiography; Hypotension; Pine Needle Extract

\section{Introduction}

In Korea, there is growing public interest in incorporating pine needle-based products because of their health promotion. Different parts of pine trees (Pinus densiflora) are consumed as foods or dietary supplements in East Asia [1,2]. Pine needle extracts (PNE) are especially known as potential anti-hypertensive [2], anti-oxidant [3, 4], anti-inflammatory [5], and anti-lipidemic agents [6]. The causes of perioperative hypotension, such as hypovolemia, myocardial depression, anesthetic overdoses, immunologic alteration, endocrine disease, allergic reaction to some antibiotics or other drugs, are very complex. We report a case of profound, long-lasting unexplained hypotension during and after anesthesia in a patient treated with PNE.

\section{Case Report}

A healthy 30-year-old woman was admitted for elective excision of a hemangioma on the right thigh. She had no relevant medical history and all laboratory findings including electrocardiogram (EKG) were within normal ranges (Figure 1). Her body weight was $50 \mathrm{~kg}$ and her height was $160 \mathrm{~cm}$. Upon arrival in the operating room, she was slightly hypotensive. Her blood pressure (BP) was $90 / 50 \mathrm{~mm} \mathrm{Hg}$ and heart rate (HR) was 80 beats $/ \mathrm{min}$. Anesthesia was induced with thiopental sodium $(200 \mathrm{mg}$ iv), midazolam ( $5 \mathrm{mg}$ iv), fentanyl (50 $\mu \mathrm{g}$ iv) and rocuronium (30 mg iv), and maintained with $\mathrm{O}_{2}-\mathrm{N}_{2} \mathrm{O}(1.5 \mathrm{~L}$ $1.5 \mathrm{~L}$ ) with $1 \%-1.5 \%$ sevoflurane. During surgery, one hour after anesthetic induction, her BP suddenly dropped to $60 / 34 \mathrm{~mm} \mathrm{Hg}$ and was not recovered in $10 \mathrm{~min}$ even

"Corresponding author. though all administered anesthetic agents were stopped immediately. Ephedrine $5 \mathrm{mg}$ iv bolus was given twice. Fifteen minutes later, the patient's BP dropped again to $65 / 37 \mathrm{~mm} \mathrm{Hg}$ and her HR increased to $150 / \mathrm{min}$. ST depression $(-2.1 \mathrm{mV})$ was noted simultaneously on lead II for a period of 2 - $3 \mathrm{~min}$. Dopamine $5-10 \mu \mathrm{g} / \mathrm{min} / \mathrm{kg}$ was infused continuously and her vital signs recovered slowly to preoperative levels. The balance of intraoperative fluid intake and output was optimal during anesthesia. In the post-anesthesia care unit (PACU) the patient was fully awake and was extubated. Slight hypokinesia of the antero-septal wall was detected in bedside transthoracic echocardiography (TTE) but there was no definite evidence of underlying myocardial ischemia or hypovolemia that may have caused the patient's intraoperative hypotension. The ejection fraction was $64 \%$. Chest X-rays and cardiac enzyme samples taken while the patient was in the PACU were all within normal limits and repeated 12-lead ECG was also normal (Figure 2). In the intensive care unit (ICU), her BP dropped again to $60 / 40 \mathrm{~mm} \mathrm{Hg}$ when dopamine was tapered and discontinued. Coronary angiography and second look TTE performed in the ICU were normal. We took another complete medical history and confirmed that the patient had no history of orthostatic hypotension, dizziness, syncope, myocardial ischemia, or endocrine disease preoperatively. However, she reported self-medication with PNE (Kwang Dong Pharmaceutical Company, Seoul, South Korea) three times per day for the three months prior to admission even without missing one time for general health promotion. The PNE from Kwang Dong Pharmaceutical Company consisted of $750 \mathrm{mg}$ of highly-concen 


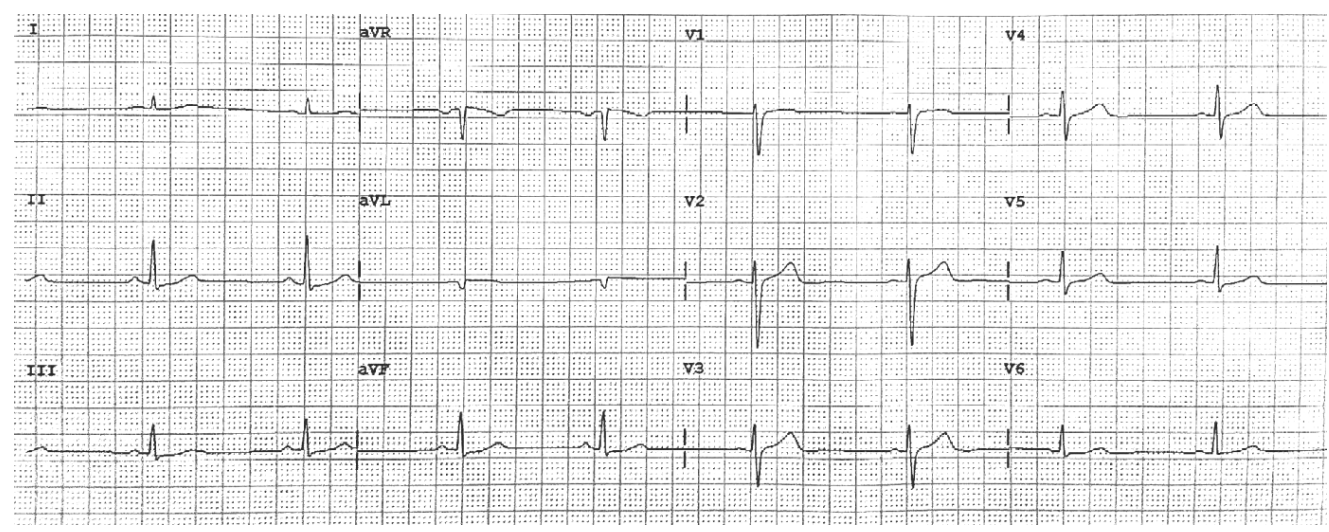

Figure 1. Preoperative electrocardiogram.

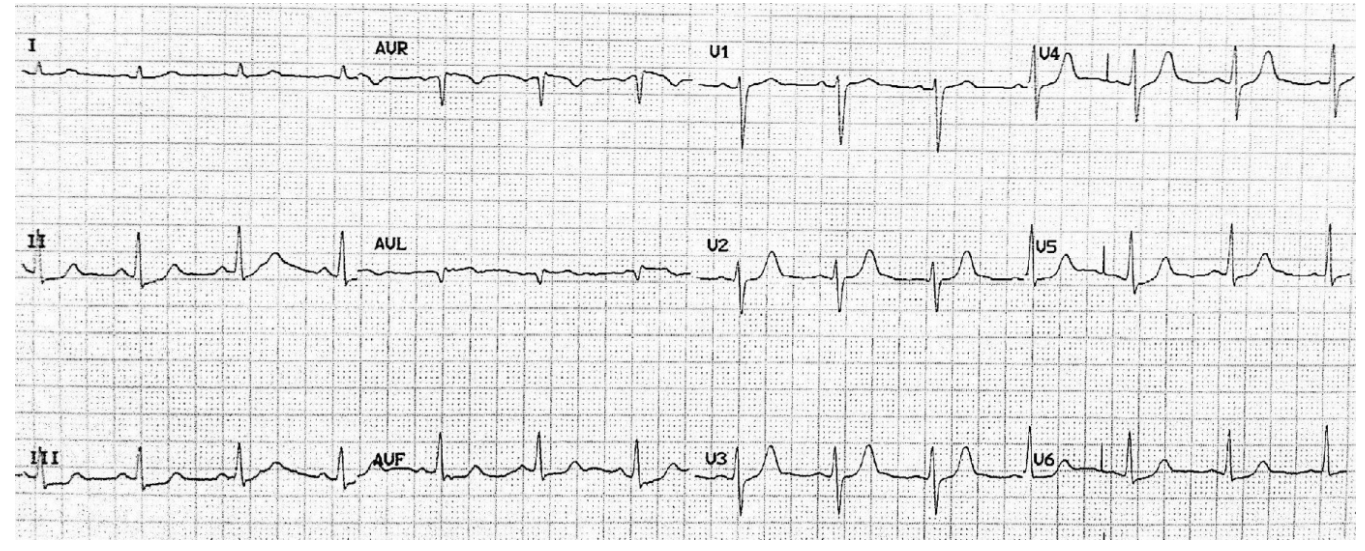

Figure 2. Electrocardiogram in post-operative care unit.

trated PNE in $120 \mathrm{ml}$ of watery solvent. The Korean Food and Drug Administration (KFDA) permitted the PNE from Kwang Dong Pharmaceutical Company as an official health promoter for the hyperlipidemia and the hyperglycemia. Dopamine (3 - $5 \mathrm{mg} / \mathrm{kg} / \mathrm{min}$ ) was infused postoperatively for $20 \mathrm{hr}$ in the ICU and finally discontinued. The patient was discharged on the third postoperative day without further hypotensive episodes. Her serum levels of cortisol and thyroid hormone at the day of operation turned out to be normal at outpatient follow up visits.

\section{Discussion}

PNE of Pinus densiflora is a popular herbal remedy in East Asia and is known to induce hypotension [2]. One of the PNE components, proanthocyanidins are pharmacologically essential, which have shown anti-hypertensive effects as well as anti-oxidative effects by repairing oxidative DNA damage and apoptosis due to hydroxyl radicals [3]. A previous study reported that the ingestion of PNE by late-gestation beef cows increased premature delivery rates $[7,8]$, perhaps due to progressively decreased uterine arterial blood flow $(>50 \%)$ caused by the direct effects of arterial smooth muscle cells or by re- duced secretion of estrogen due to low uterine blood flow. Recently another reported that PNE directly inhibited contractile responses of isolated strips of rat aorta and induced significant hypotension in normotensive and phenylephrine-challenged hypertensive rats [9].

The causes of intraoperative hypotension are multifactorial. Patients under anesthesia and undergoing surgery may experience hypovolemia caused by fluid deficits. In our case, TTE in the PACU showed appropriate left ventricular end-diastolic volume (LVEDV) and ejection fraction (EF), so intraoperative hypovolemia can be ruled out. Myocardial depression caused by angina or myocardial ischemia may also cause intraoperative hypotension. In our patient, the preoperative EKG was normal and serum triglycerides and low density lipoprotein cholesterol values were within normal ranges. There was no positive history of chest discomfort, epigastric pain, or impairment of physical activities. Intraoperative EKG showed tachycardia (up to $150 \mathrm{beat} / \mathrm{min}$ ) and ST depression $(-2.1 \mathrm{mV})$ suggesting reversible transient subendocardial infarction (2 - $3 \mathrm{~min})$, but such findings do not precede hypotensive insults of the myocardium. TTE taken in the PACU and ICU did not reveal wall motion abnormality or hypokinesia and the patient's cardiac 
enzymes were all within normal limits, so intraoperative myocardial ischemia preceding hypotension could be excluded. Hypotension may also be caused by anesthetic overdose. Abrupt hypotension occurred 1 hour after anesthetic induction in our patient. Most anesthetic induction agents are expected to be metabolized and eliminated by that time. When our patient became hypotensive, all gases including sevoflurane $(-1$ vol $\%$ in end tidal concentration) and nitrous oxide $\left(\mathrm{N}_{2} \mathrm{O}\right)$ were discontinued immediately. BP and HR were elevated in response to the intravenous administration of ephedrine $10 \mathrm{mg}$, but dropped in $15 \mathrm{~min}$ to $64 / 37 \mathrm{~mm} \mathrm{Hg}$ even though all inhalational anesthetics were discontinued. Continuous dopamine infusion was the only way to maintain intraoperative and postoperative BP in our patient, so anesthetic overdose may be ruled out. Echocardiography is a valuable noninvasive diagnostic tool for the investigation of intraoperative hypotension. Contractility and volume status of the left ventricle can be easily and immediately measured, and other important causes of hypotension are easily diagnosed, such as cardiac tamponade or aortic aneurysms $[10,11]$.

There are so many factors that affect the blood pressure of the patient perioperatively. Even we tried to exclude the major reasons of perioperative hypotension, there was no definite proofs in this situation. Also we could not exclude the possibilities of parasympathetic over-reactivity during the procedures, related to laryngoscopy, stretching nerves of thigh, and administration of reversal drug (pyridostigmine $10 \mathrm{mg}$ ) of neuromuscular blocker. We assume that meticulous administration of PNE may induce the direct vasodilation of systemic arteries in our patient from the similar mechanism of previous reports in vivo [7,8] and in vitro [9]. Superimposed anesthetic administration may worsen the vasodilatory phenomenon of our PNE-medicated patient.

Briefly, there is growing demand for herbal remedies such as PNE, especially in East Asia. PNE is known to induce profound, long-lasting hypotension that is selflimiting but can produce unexplained perioperative hypotension that is intractable to intermittent vasopressor injection. Therefore, ischemic insults of the myocardium are possible in patients who take PNE. In our experience, complete history-taking and insight of preoperative administration of herbal remedies are important. Supportive cares for long-lasting hypotension should be provided with infusion of continuous vasopressor. To exclude other causes of perioperative hypotension, immediate diagnostic evaluation including bedside echocardiography and coronary angiography are essential in PNE-medicated patients for safe anesthetic management.

\section{REFERENCES}

[1] N. Y. Kim, M. K. Jang, D. G. Lee, K. H. Yu, H. J. Jang, M. Kim, S. G. Kim, B. H. Yoo and S. H. Lee, "Comparison of Methods for Proanthocyanidin Extraction from Pine (Pinus densiflora) Needles and Biological Activities of Extracts," Nutrition Research and Practice, Vol. 4, No. 1, 2010, pp. 16-22. doi:10.4162/nrp.2010.4.1.16

[2] K. Y. Kim and H. J. Chung, "Flavor Compounds of Pine Sprout Tea and Pine Needle Tea," Journal of Agricultural and Food Chemistry, Vol. 48, No. 4, 2000, pp. 1269-1272. doi:10.1021/jf9900229

[3] J. B. Jeong, E. W. Seo and H. J. Jeong, "Effects of Extracts of Pine Needle against Oxidative DNA Damage and Apoptosis Induced by Hydroxyl Radical via Antioxidant Activities," Food and Chemical Toxicology, Vol. 47, No. 8, 2009, pp. 2135-2141. doi:10.1016/j.fct.2009.05.034

[4] M. J. Jung, H. A. Jung, S. S. Kang, G. S. Hwang and J. S. Choi, "A New Abietic Acid-Type Diterpene Glucoside from the Needles of Pinus densiflora," Archives of Pharmacal Research, Vol. 32, No. 12, pp. 1699-1704.

[5] J. H. Kwon, J. H. Kim, S. E. Choi, K. H. Park and M. W. Lee, "Inhibitory Effects of Phenolic Compounds from Needles of Pinus densiflora on Nitric Oxide and $\mathrm{PGE}_{2}$ Production," Archives of Pharmacal Research, Vol. 33, No. 12, 2010, pp. 2011-2016. doi:10.1007/s12272-010-1217-y

[6] J. R. Jeon and J. Y. Kim, "Effects of Pine Needle Extract on Differentiation of 3T3-L1 Preadipocytes and Obesity in High-Fat Diet Fed Rats," Biological \& Pharmaceutical Bulletin, Vol. 29, No. 10, 2006, pp. 2111-2115. doi:10.1248/bpb.29.2111

[7] S. P. Ford, L. K. Christenson, J. P. Rosazza and R. E. Short, "Effects of Ponderosa Pine Needle Ingestion on Uterine Vascular Function in Late-Gestation Beef Cows," Journal of Animal Science, Vol. 70, No. 5, pp. 16091614.

[8] F. C. Chow, K. J. Hanson, D. W. Hamer and R. H. Udall, "Reproductive Failure of Mice Caused by Pine Needle Ingestion," Journal of Reproduction and Fertility, Vol. 30, No. 1, 1972, pp. 169-172. doi:10.1530/jrf.0.0300169

[9] H. S. Cheong and D. Y. Lim, "Pine Needle Extracts Inhibit Contractile Responses of the Isolated Rat Aortic Stripes," Natural Product Sciences, Vol. 16, No. 2, 2010, pp. 123-132.

[10] B. Subramaniam and D. Talmor, "Echocardiography for Management of Hypotension in the Intensive Care Unit," Critical Care Medicine, Vol. 35, No. 8, 2007, pp S401S407. doi:10.1097/01.CCM.0000270277.29661.3B

[11] B. S. Cowie, "Focused Transthorasic Echocardiography in the Perioperative Period," Anaesth Intensive Care, Vol. 38, No. 5, 2010, pp. 823-836. 